\title{
Tilt, polarity, and spontaneous symmetry breaking in liquid crystals
}

\author{
Alexandros G. Vanakaras and Demetri J. Photinos \\ Department of Physics, University of Patras, Patras 26500, Greece \\ Edward T. Samulski \\ Department of Chemistry, University of North Carolina, Chapel Hill, North Carolina 27599-3290
}

(Received 12 January 1998)

\begin{abstract}
We show through explicit molecular modeling how tilt may be induced in layered mesophases and propagate across the layers, using a concrete representation of flexible tail-core-tail calamitic mesogens in conjunction with the variational cluster expansion. The results demonstrate that spontaneous symmetry breaking observed in smectic liquid crystals - the tilt of the director relative to the layers - can be induced by excluded volume interactions, both in the synclinic and in the anticlinic configurations. [S1063-651X(98)51105-1]
\end{abstract}

PACS number(s): 61.30.Cz, 64.70.Md

The relationship between molecular symmetry and phase symmetry in liquid crystals is very intriguing. The nematic phase - a fluid of orientationally ordered mesogens-is apolar even in cases where the mesogen itself is polar, either in its shape or its electric charge distribution [1]. By contrast, stratified smectic liquid crystals_-orientationally ordered fluids wherein the mesogen centers of mass condense into layers - exhibit polarity even when the molecules are apolar; in a tilted smectic- $C\left(S_{C}\right)$ phase comprised of apolar mesogens, the phase is polar-the " $C$ director' specifying the tilt direction, is a unique direction in the $S_{C}$ phase [1]. Additionally, polarity normal to the "tilt plane" is indigenous to the $S_{C}$ phase even when the latter is comprised of apolar mesogens [2]. Thus the relationship between molecular symmetry and phase symmetry appears ambiguous in the sense that the latter cannot always be inferred from the former. In particular, there are instances where the apparent (local) symmetry of the phase is lower than that of its constituent molecules, a feature that is emblematic of so-called spontaneous symmetry breaking (SSB).

In this Rapid Communication we focus on attributes of the orientational ordering of flexible molecules in the normal $S_{A}$ and tilted $S_{C}$ phases by starting out with an explicit molecular structure-a generic, three-segment model of a mesogen - and performing calculations using a well-defined statistical-mechanical approximation, the variational cluster expansion [3]. We obtain clear insights into factors that influence the tilt in smectics, the propagation of the tilted ordering across the smectic layers (synclinic vs anticlinic), and the sign and magnitude of the spontaneous polarization in the chiral smectic- $C\left(S_{C}^{*}\right)$ phase. Our findings constitute an explicit derivation of the fundamental propositions of the "indigenous polarity (IP) theory' of Photinos and Samulski $[2,4]$ and provide a general conceptual framework for understanding polarity and ferroelectricity across the entire spectrum of liquid crystals, in calamitic, discotic, and bent ("banana-" or "boomerang-shaped") mesogens.

The first efforts to derive the structure of the tilted achiral $S_{C}$ phase from a molecular model date back to the theory of McMillan [5] in which the tilt is a result of end-to-end alignment of permanent dipoles attached obliquely with respect to the mesogen's "'long-molecular-axis"' $l$. The McMillan model, and variants thereof that followed [6], imply that the appearance of director tilt in the $S_{C}$ phase is inevitably accompanied by strong biasing of the rotational motion about $l$ (partial rotational freeze-out) [7]. But this picture is in direct conflict with experimental results. In fact, obtaining tilt at the price of rotational biasing undermines rigid-molecule models that have been proposed for the description of spontaneous polarization in tilted smectics [8].

Herein we study the SSB mechanism associated with tilted smectics using idealized, three-segment, model mesogens - a primitive representation of flexible tail-coretail calamitic mesogens (Fig. 1). For simplicity we consider two-dimensional phases, in the $Y Z$ plane, with perfect smectic layering in the $Z$ direction, i.e., the molecular centers are assumed to be confined on "smectic lines", of length $L$ and constant spacing $d$. This precludes the description of positional fluctuations in the direction of the layer normal. It is justified, however, in that such fluctuations are not of primary relevance to the SSB mechanism.

Molecular flexibility is imparted to the model, while

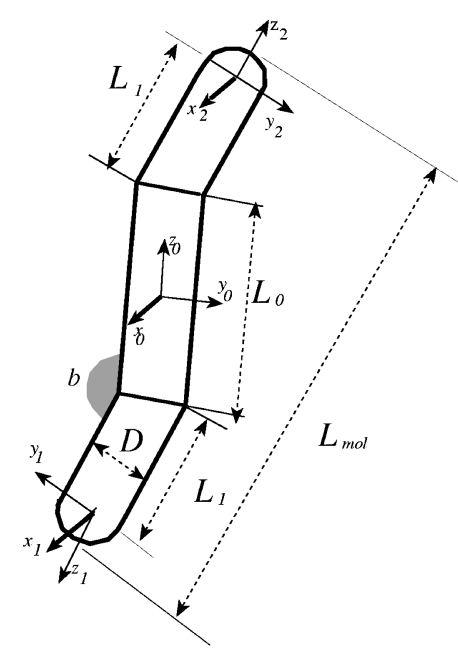

a
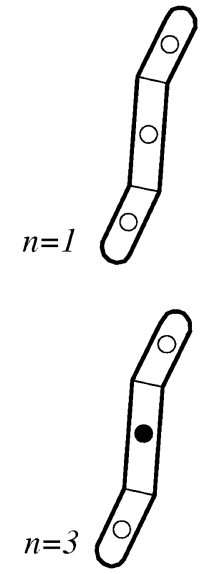

b
FIG. 1. (a) Schematic diagram of the three-segment molecular model, showing the segmental axis frames $x_{i}, y_{i}, z_{i}$, the segmental width $D$ and lengths $L_{i}$, the total molecular length $L_{m o l}$, and the core-tail linkage angle $b$. (b) Four distinct planar configurations $(n=1-4)$ of a tail-core-tail mesogen. The closed and open circles indicate the two opposite orientations of the segmental axes $x_{i}$. 
maintaining molecular planarity, by allowing the tails to perform concerted $180^{\circ}$ flips about the core axis, thereby generating four planar and intrinsically equiprobable "conformations" shown in Fig. $1(n=1-4)$. The orientation of each conformer with respect to the $Y Z$ frame is then completely specified by the angle $\alpha$ of the core-segment axis with the $Z$ axis. As a result, the intermolecular potential $u_{n, n^{\prime}}^{s, s^{\prime}}$, for a pair of molecules in configurations $n, n^{\prime}$ and situated on the smectic lines $s, s^{\prime}$, depends on the coordinate difference $Y-Y^{\prime}$ and the angles $\alpha, \alpha^{\prime}$.

The molecules are assumed to interact exclusively through hard body repulsions. In this case the molecules are obviously apolar, achiral, and they possess three planes of (statistical) symmetry, which can be identified with the $x_{0} y_{0}$, $x_{0} z_{0}$, and $y_{0} z_{0}$ planes of the molecular frame attached to the core segments. Furthermore, it is noted that the molecular conformers with $n=1$ and 3 are identical with respect to their interactions (and so are the ones with $n=2,4$ ). These conformers differ in that they correspond to opposite directions of the $x_{0}$ and $y_{0}$ axes of the core frames of reference. Such sign reversals have no bearing on the interaction, but their distinction is needed for the description of the polar ordering of the molecular segments.

In the two-molecule cluster approximation, the free energy $F^{(2)}$ of a such a system, consisting of $N$ molecules at temperature $T$, is given by the expression

$$
\frac{F^{(2)}}{N k T}=\ln \rho-1-\ln \zeta-\frac{1}{2} \rho_{L}^{*}\left\langle v_{n}^{s}(\alpha)\right\rangle,
$$

where $\rho=N / L d$ denotes the molecular number density and $\rho_{L}^{*}$ is the effective linear density of molecules in the smectic lines [9]. The quantity denoted by $v_{n}^{s}(\alpha)$ is the effective single-molecule potential; it is obtained self-consistently from the interaction potential according to the relation

$$
v_{n}^{s}(\alpha)=\frac{1}{L} \sum_{s^{\prime}, n^{\prime}} \int d \alpha^{\prime} P_{n^{\prime}}^{s^{\prime}}\left(\alpha^{\prime}\right) \int d Y^{\prime} f_{n, n^{\prime}}^{s, s^{\prime}}\left(\alpha, \alpha^{\prime} ; Y-Y^{\prime}\right),
$$

where $f_{n, n^{\prime}}^{s, s^{\prime}}=1-\exp \left(-u_{n, n^{\prime}}^{s, s^{\prime}} / k T\right)$. Due to the assumed hardbody interactions, $f_{n, n^{\prime}}^{s, s^{\prime}}=1$ if the molecules intersect and $f_{n, n^{\prime}}^{s, s^{\prime}}=0$ otherwise. The effective potential determines the single-molecule distribution function $P_{n}^{s}(\alpha)$ :

$$
P_{n}^{s}(\alpha)=\frac{1}{\zeta} \exp \left[-\rho_{L}^{*} v_{n}^{s}(\alpha)\right]
$$

where the normalization factor $\zeta$ is given by

$$
\zeta=\sum_{s, n} \int d \alpha \exp \left[-\rho_{L}^{*} v_{n}^{s}(\alpha)\right]
$$

The angular brackets in Eq. 1 indicate ensemble averaging with respect to the single particle distribution of Eq. (3).

Equations 2 and 3 establish the required connection of the single-molecule distribution to the intermolecular potential. Given the latter, Eq. (2) is solved for $v_{n}^{s}(\alpha)$ at fixed linear density. The relative thermodynamic stability of the phases described by the different solutions is determined by comparing the respective values of the free energy. The layer spacing is determined, as a function of the (overall) molecular number density, by further minimizing the free energy with respect to $d$.

There are three types of solutions that describe smectic phases with disorder of the molecular positions within the layers [10]:

(a) Smectic-A solutions. These correspond to $v_{n}^{s}(\alpha)$ invariant with respect to translations across the layers (i.e., independent of $s$ ) and with respect to "rotations" (180 flips, in this two-dimensional case) of the molecular orientations about the layer normal. For given spacing $d$, the solutions describing the $S_{A}$ phase can be obtained for any value of $\rho_{L}$. The orientational order parameters $\langle\cos 2 m \alpha\rangle(m$ $=1,2,3, \ldots)$ are nonvanishing, whereas $\langle\sin 2 m \alpha\rangle=0$ due to the twofold symmetry about the $Z$ axis. The same symmetry restrictions apply to the segmental order parameters for the tail segments.

(b) Smectic-C solutions with uniform, synclinic tilt. These correspond to $v_{n}^{s}(\alpha)$ invariant with respect to translations across the layers but not symmetric with respect to flips of the molecular orientations about the layer normal. Here the twofold symmetry about the $Z$ axis (and about the $Y$ axis) is broken and this is manifested by the order parameters $\left\langle\sin 2 m \alpha_{i}\right\rangle, m=1,2, \ldots$, developing nonvanishing values as the density exceeds a certain critical value. These order parameters grow continuously from zero, resulting in secondorder transitions from the $S_{A}$ phase to the $S_{C}$. The solutions present a twofold degeneracy; for each solution $v_{n}^{s}(\alpha)$ there is a second, distinct solution, obtained by twofold rotation about the $Z$ axis, and resulting in identical values for the free energy. This reflects the spontaneous character of the symmetry breaking or, alternatively, the preservation of the intrinsic symmetry of the molecular system about the $Z$ axis, albeit in a global sense; the system has equal probability to assume either of the two (distinct, but symmetry related) possibilities of molecular self-organization.

In addition to the onset of tilted ordering, the SSB is accompanied by another, equally profound, qualitative change of molecular self-organization; the $x$ axis of the tail segments develops polar ordering along the $X$ axis of the phase, i.e., the order parameters $C_{x_{i} X}=\left\langle\mathbf{x}_{i} \cdot \mathbf{X}\right\rangle$, for $i=1,2$ are nonvanishing. This transverse polarity of the smectic layers is the combined result of the tilting and of the bent disposition of the tail segments relative to the core axis $[2,4]$.

(c) Smectic-C solutions with constant magnitude, anticlinic tilt. These correspond to $v_{n}^{s}(\alpha)$ alternating with $s$ between two forms, each of which is asymmetric (about the layer normal), and is related to the other according to $v_{n^{+}}^{s}\left(\alpha^{+}\right)=v_{n^{-}}^{s^{+}}\left(\alpha^{-}\right)$, where $n^{ \pm}, \alpha^{ \pm}$denote molecular configurations that are obtained from each other by $180^{\circ}$ flips about the layer normal. The corresponding free energy is never found to be lower than that of the synclinic $S_{C}$ phases, although the difference is normally rather small and vanishes when the layer spacing reaches its maximal value $d=L_{m o l}$. The individual anticlinic smectic layers exhibit polarity in the ordering of the $x_{i}$ axes of the tail segments, but with alternating sign of the order parameter in moving from one layer to the next. Unlike the synclinic case, the $Y$ axis is a twofold symmetry axis for any pair of successive layers in the anticlinic $S_{C}$ phase. 

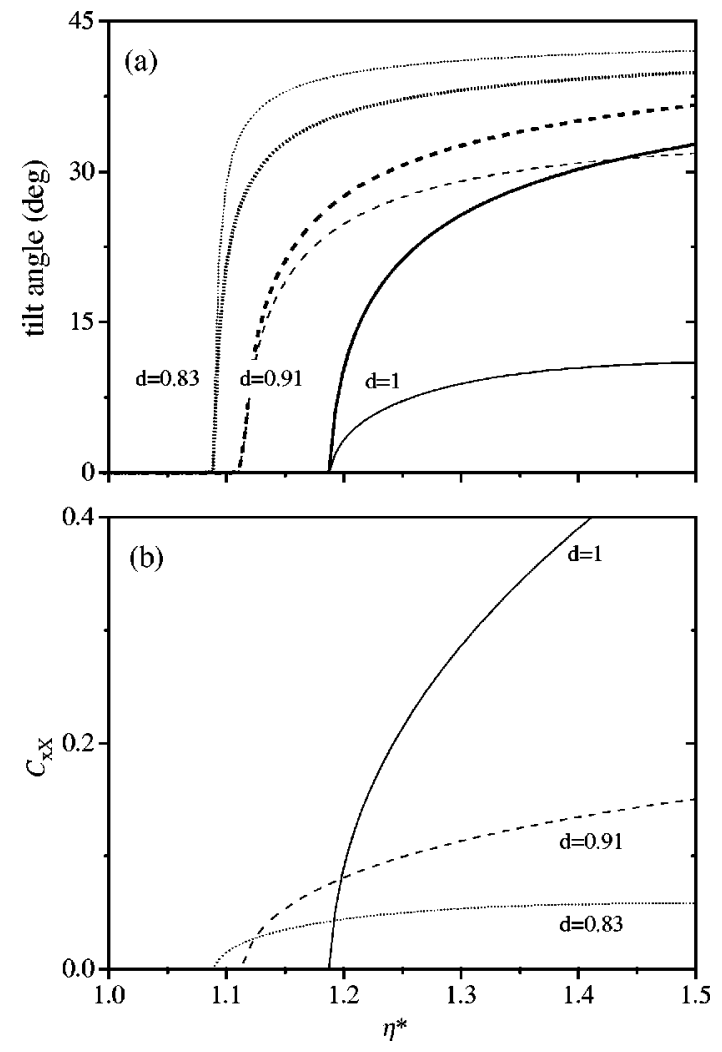

FIG. 2. (a) Plots of the density dependence of the tilt angles of the core (thick lines) and tail segments (thin lines), for various values of the layer spacing $d$ (in units of the molecular length $\left.L_{m o l}\right)$. The results are for molecules with $L_{0} / D=5, L_{1}=L_{2}$ $=L_{0} / 2$, and core-tail linkage angle $b=145^{\circ}$. The density is expressed in terms of the effective packing fraction $\eta^{*}$ $=\left(2 L_{0} D / d\right) \rho_{L}^{*}$. (b) The respective plots of the density dependence of the polar order parameters $C_{x_{i} X}$ of the tail segments.

The results of the calculations for representative values of the molecular geometry parameters, and for selected values of the layer spacing, are plotted in Fig. 2. Due to the nonlinear and flexible structure of the molecules, the tilt angle for the core segment is, in general, different from that of the tails [2]. The plots in Fig. 2(a) show that the core segments are more tilted than the tails for $d=L_{m o l}$. As $d$ decreases, the tilt angle of the tails increases more rapidly and eventually exceeds that of the core. The polar order parameter $C_{x_{i} X}$ is seen to decrease continuously with decreasing layer spacing. This parameter undergoes a sign reversal as the spacing decreases below a certain crossover value, which depends on the linkage angle $b$ and the $L_{i} / L_{0}$ ratios.

The calculations show that purely hard-body repulsions are sufficient to generate the tilted smectic ordering and transverse polarity. Localized interactions that had been proposed in previous models $[5,6,8]$ of the $S_{C}$ phase (of the dipole-dipole, dipole-induced dipole, quadrupole-quadrupole types, etc.) are not necessary. Furthermore, the molecules need not exhibit monoclinic asymmetry; the SSB mechanism makes it possible for the flexible molecules to attain tilted smectic ordering even when their statistical symmetry is higher (as is the case with many real smectogens) than the symmetry of the resulting phase. Finally, the free-energy difference between the synclinic and anticlinic ordering is, in general, small (especially when the layer spacing is not much

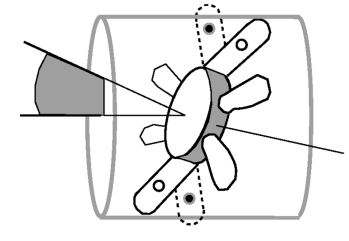

a

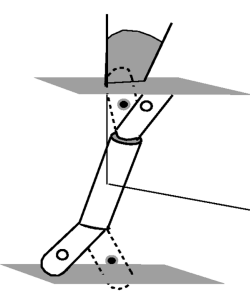

b

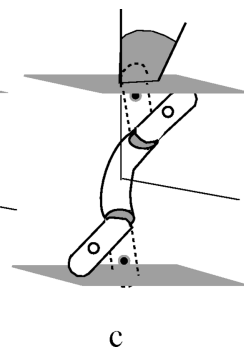

FIG. 3. The mesogenic cores and tail segments are schematically illustrated for discotic (a), calamitic (b), and (c) bent banana mesogens, to emphasize the similarity of all three types of mesogens with respect to the packing constraints that give rise to tilt and polar ordering. The spontaneous symmetry breaking (tilt with respect to the column axis or layer normal) shifts the probability distribution towards configurations with one sense of tail-tranverse polarity. The polarity is tangent to the disk plane, and along the respective $C 2$ axes of the calamitic and bent mesogens.

smaller than the molecular length). Therefore, additional interactions that are otherwise subtle (such as dipole-dipole interactions and the resulting flexibility of the tail segments) could readily shift the thermodynamic stability in favor of one type of arrangement or the other.

Minimization of the free energy with respect to the layer spacing (at constant overall density $N / V$ ) shows that the value $d=L_{m o l}$ gives the thermodynamically most stable layer spacing within the density regime of positionally disordered smectic phases. This is not surprising in view of the purely hard-body interactions used in the calculation. It can be shown, however, that the inclusion of symmetric "soft" interactions is sufficient to stabilize the denser layering.

While the present calculations pertain to the threesegment model, an idealization of the shape adopted in actual flexible calamitic mesogens, the essential findings can be generalized conceptually to account for observations in discotic [11] and bent [12] mesogens. Consider the relationship between the idealized "three-segment" representations of all three kinds of mesogens in Fig. 3. For all three, packing constraints can induce a SSB transition and indigenous polarity will be manifested. In the case of the discotic mesogen, the IP-and its spontaneous polarization, if the tails are dipolar and chiral-is tangent to the disk core and normal to the columns. In the case of the bent mesogen, "head-to-tail', packing of $V$-shaped objects in a "line" $(>>>)$ is inherently polar. Contrary to previous suggestions [13], this polarity is not a consequence of a SSB transition; it is a consequence of the asymmetry inherent in the $V$-shaped mesogen. However, one can induce SSB (tilt) in phases comprised of $V$-shaped molecules and associated indigenous polarity when the conformer distribution of the tails is perturbed by packing constraints of the type we maintain is operative in calamitic smectics. In summary we conclude that excluded volume interactions within the strata of smectics and within columnar phases constitute a general microscopic mechanism for affecting SSB (tilt) and indigenous polarity (and spontaneous polarization) in liquid crystals.

This work was supported by the National Science Foundation (Grant No. DMR-9412701). 
[1] P. G. de Gennes and J. Prost, The Physics of Liquid Crystals, 2nd ed. (Oxford University Press, London, 1993).

[2] D. J. Photinos and E. T. Samulski, Science 270, 783 (1995).

[3] For the application of this method to the description of liquidcrystal phases and its relation to the mean-field approach, the Onsager approach, and the density-functional approach, see A. G. Vanakaras and D. J. Photinos, Mol. Phys. 85, 1089 (1995), and references therein.

[4] D. J. Photinos, A. F. Terzis, E. T. Samulski, T. J. Dingemans, A. Chen, and C-D. Poon, Mol. Cryst. Liq. Cryst. Sci. Technol., Sect. A 292, 265 (1997); A. F. Terzis, D. J. Photinos, and E. T. Samulski, J. Chem. Phys. 107, 4061 (1997).

[5] W. L. Mc Millan, Phys. Rev. A 8, 1921 (1973); R. J. Meyer and W. L. McMillan, ibid. 9, 899 (1974).

[6] For a discussion of various models of the $S_{C}$ phase see, for example, J. W. A. Goosens, Phys. Rev. A 40, 4019 (1989); J. Phys. (France) 46, 1411 (1985); G. Vertogen and W. H. de Jeu, Thermotropic Liquid Crystals, Fundamentals (SpringerVerlag, New York, 1988); A. Poniewierski and T. J. Sluckin, Mol. Phys. 73, 199 (1991).

[7] Restricted rotation is not a prerequisite for tilt; Priest pointed out that even for rigid, axially symmetric molecules, tilt is a theoretically viable possibility [R. G. Priest, J. Chem. Phys. 65, 408 (1976)].
[8] See, for example, M. A. Osipov, and S. A. Pikin, J. Phys. II 5, 1 (1995); B. Kutnjak-Urbanc and B. Zeks, Liq. Cryst. 18, 483 (1995).

[9] When higher-order cluster contributions are completely ignored, $\rho_{L}^{*}$ reduces to the actual linear density $N_{L} / L$, where $N_{L}$ is the number of molecules per linear smectic layer. It is possible, however, to partially account for such contributions [see, for example, S.-D. Lee, J. Chem. Phys. 87, 4972 (1987)], in which case $\rho_{L}^{*}$ becomes a more complex function of the actual linear density and the whole procedure reduces to a density rescaling, which is of no qualitative significance to the present results.

[10] In all cases the orientational ordering within each layer exhibits twofold rotational symmetry $(C 2)$ about the $X$ axis. Consequently, the odd rank order parameters $\langle\cos (2 m+1) \alpha\rangle$ and $\langle\sin (2 m+1) \alpha\rangle, m=1,2, \ldots$, vanish.

[11] H. Bock and W. Helfrich, Liq. Cryst. 18, 387 (1995); G. Scherowsky and X. H. Chen, Mater. Chem. Phys. 5, 417 (1995).

[12] T. Noiri, T. Sekine, J. Watanabe, T. Furukawa, and H. Takezoe, J. Mater. Chem. 6, 1231 (1996).

[13] D. R. Link, G. Natale, R. Shao, J. E. Maclennan, N. A. Clark, E. Korblova, and D. M. Walba, Science 278, 1924 (1997). 